\title{
A stochastic order for the analysis of investments affected by the time value of money
}

\author{
M.C. López-Díaz, M. López-Díaz ${ }^{b, *}$, S. Martínez-Fernández ${ }^{c}$ \\ ${ }^{a}$ Departamento de Matemáticas, Universidad de Oviedo. C/Calvo Sotelo s/n. \\ E-33007 Oviedo, Spain. e-mail: cld@uniovi.es \\ ${ }^{b}$ Departamento de Estadística e I.O. y D.M., Universidad de Oviedo. C/Calvo \\ Sotelo s/n. E-33007 Oviedo, Spain. e-mail: mld@uniovi.es \\ ${ }^{c}$ Unidad de Modelos de Riesgos, Liberbank. Plaza de La Escandalera, 2. E-33003 \\ Oviedo, Spain. e-mail: smartinezf@liberbank.es
}

\begin{abstract}
Time value of money reads that an amount of money at the present time is worth more than the same amount in future because its earning capacity and inflation. This fact is reflected in multiple financial concepts and in the final result of numerous investments by means of functions which satisfy appropriate properties. Motivated by the need to compare such investments we introduce an integral stochastic order generated by those functions. The maximal generator of the order is obtained. It is proved that the new stochastic order is generated by a non-stochastic partial order and the class of preserving mappings of such a partial order. Characterizations of the order are developed. Relevant properties, as well as connections with other stochastic orderings and examples, are studied.
\end{abstract}

JEL code: G11 Portfolio Choice; Investment Decisions

Keywords: cash flow, maximal generator, stochastic order, time value of money

\footnotetext{
${ }^{*}$ Corresponding author.
} 


\section{Motivation}

Time value of money says that a quantity of money today is more valuable than the same quantity later on. This is because we can handle money to make more money with investments, and because inflation reduces the power of the money, thus modifying its value.

Time value of money is a fundamental idea in financial management (see, for instance, Crosson and Needles (2010), page 442, and Tretyakov (2013), page 10). There is a broad diversity of economic concepts which reflect that idea, like the present value, the present value of an annuity, the present value of a perpetuity, the net present value, the future value, the future value of an annuity, etc. Problems in relation to this concept concern the net value of cash flows at different moments in time (see, for instance, De Schepper et al. (2002) or Dhaene et al. (2012)).

Long-term investments are affected by the time value of money. Mathematical expressions for the study of those investments show the aforementioned fact. Assume an investment where $x_{i}$ stands for the annual cash flow at the end of the $i^{\text {th }}$ year after its beginning. The analysis of such an investment when $n$ years have passed, is frequently based on the value $f\left(x_{1}, x_{2}, \ldots, x_{n}\right)$, where the mapping $f: \mathbb{R}^{n} \rightarrow \mathbb{R}$ represents the profitability and satisfies both $f\left(x+\varepsilon e_{i}\right) \geq f\left(x+\delta e_{i+1}\right), 1 \leq i \leq n-1, e_{i}$ being the $i^{t h}$-unit vector $(0, \ldots, 0,1,0, \ldots, 0)$, number 1 in position $i, 0 \leq \delta \leq \varepsilon$, $x=\left(x_{1}, x_{2}, \ldots, x_{n}\right) \in \mathbb{R}^{n}$, and $f$ is increasing.

The first condition of $f$ means that it is preferable an amount of money in the $i^{t h}$ year than a non-greater amount in the following year (time value of money). The meaning of the second condition is clear, the greater the money we have, the better the final result of our investment.

Let us show examples of financial investments under that framework.

Suppose that an initial capital is invested in a business whose positive cash flow at the end of the $i^{t h}$ year is $x_{i}$, and that cash flow is invested at annual interest rates $r_{j}$ in subsequent years $j$. The amount of money obtained with the investment after $n$ years is $x_{1}\left(1+r_{2}\right) \ldots\left(1+r_{n}\right)+x_{2}\left(1+r_{3}\right) \ldots\left(1+r_{n}\right)+$ $\ldots+x_{n-1}\left(1+r_{n}\right)+x_{n}$. Such an amount is given by a mapping satisfying the above two conditions, in this case $f\left(x_{1}, x_{2}, \ldots, x_{n}\right)=\sum_{i=1}^{n}\left(\prod_{j=i+1}^{n}\left(1+r_{j}\right)\right) x_{i}$.

Assume a second business to invest the initial capital, whose positive annual cash flow at the end of the $i^{t h}$ year is denoted by $y_{i}$. The second business will be preferred to the former when $\sum_{i=1}^{n}\left(\prod_{j=i+1}^{n}\left(1+r_{j}\right)\right) x_{i} \leq$ $\sum_{i=1}^{n}\left(\prod_{j=i+1}^{n}\left(1+r_{j}\right)\right) y_{i}$ (note that the above formula divided by $\prod_{j=1}^{n}\left(1+r_{j}\right)$ 
leads to the net present value of the investments). If $x$ stands for the vector $\left(x_{1}, \ldots, x_{n}\right)$ and $y$ denotes the vector $\left(y_{1}, \ldots, y_{n}\right)$, an investor should compare $f(x)$ and $f(y)$.

Let us see another example. That is based on the so-called internal rate of return. Consider an investment whose initial capital is $A$. Let $x_{1}, x_{2}, \ldots, x_{n}$ be the cash flows from that investment at the end of the next $n$ years.

The internal rate of return of the investment is defined as the discount rate $r$ which makes the net present value of the investment equal to zero, that is, $r$ should satisfy $0=-A+\frac{x_{1}}{1+r}+\frac{x_{2}}{(1+r)^{2}}+\ldots+\frac{x_{n}}{(1+r)^{n}}$ (the reader is referred for instance to Brealey et al. (2014) for economic concepts).

Frequently, investments are compared by the internal rate of return, an investment is preferred to a second investment when the former has a greater internal rate of return. In order to guarantee the existence of a positive value of $r$, it is assumed that $\sum_{i=1}^{n} x_{i}>A$.

Consider a 2-years investment with $A>0$ and $x_{1}+x_{2}>A$. The internal rate of return is the value $r$ with $0=-A+\frac{x_{1}}{1+r}+\frac{x_{2}}{(1+r)^{2}}$. It is not hard to prove that $r=\frac{x_{1}+\sqrt{x_{1}^{2}+4 A x_{2}}}{2 A}-1$, and such a value is strictly positive since $x_{1}+x_{2}>A$. The internal rate of return of a 2 -years investment is

$$
r=f\left(x_{1}, x_{2}\right)= \begin{cases}\frac{x_{1}+\sqrt{x_{1}^{2}+4 A x_{2}}}{2 A}-1 & \text { if } x_{1}+x_{2}>A, \\ 0 & \text { if } x_{1}+x_{2} \leq A .\end{cases}
$$

Observe that when $x_{1}+x_{2} \leq A$, the profitability of the investment could be negative, and even there could not be a solution of $r$. Generally, those investments are dismissed, and so their internal rates of return are defined as 0 .

The comparison of 2-years investments by means of internal rates of return requires the comparison of those mappings. It can be seen that these maps satisfy the conditions considered at the beginning of this section.

Mathematical formulas for most of the problems affected by the time value of money involve functions which satisfy the conditions previously mentioned.

Cash flows generated by investments are mostly random instead of deterministic. If the random vectors $\mathbf{X}=\left(X_{1}, \ldots, X_{n}\right)$ and $\mathbf{Y}=\left(Y_{1}, \ldots, Y_{n}\right)$ represent the annual cash flows from two $n$-years investments, the analysis of those investments requires the comparison of $E(f(\mathbf{X}))$ and $E(f(\mathbf{Y}))$, where $f$ satisfies the aforementioned conditions because of time value of money, and $E$ stands for the expectation. 
In this paper we introduce a stochastic order to compare $n$-year investments affected by the time value of money. That order is integral, the class of functions $f: \mathbb{R}^{n} \rightarrow \mathbb{R}$ with $f\left(x+\varepsilon e_{i}\right) \geq f\left(x+\delta e_{i+1}\right), 1 \leq i \leq n-1,0 \leq \delta \leq \varepsilon$, $x \in \mathbb{R}^{n}$, and $f$ increasing, being a generator of the order. The maximal generator of that integral stochastic order is obtained. We prove that the new order is in fact a stochastic order generated by a (non-stochastic) partial order on $\mathbb{R}^{n}$, and the class of preserving mappings of that partial order. This permits to develop important characterizations and properties of the proposed stochastic order. Connections with other stochastic orders related to random cash flows and examples are also studied.

The structure of the paper is as follows: after this introductory section, we include the concepts and notations needed for the development of the paper in Section 2, while the results of the paper are described in Section 3. This section is divided in different subsections. Subsection 3.1 is devoted to analyze characterizations of the order and to obtain the maximal generator. Subsection 3.2 is dedicated to relevant properties of the order. Connections with other orders and examples are stated in Subsection 3.3. Conclusions are presented in Section 4.

\section{Preliminaries}

Some concepts in relation to the theory of ordered sets are necessary for the development of the paper.

Let $\mathcal{X}$ be a set. A binary relation $\preceq$ on $\mathcal{X}$ which is reflexive and transitive is said to be a pre-order. A binary relation $\preceq$ on $\mathcal{X}$ which is reflexive, transitive and antisymmetric is called a partial order. In the last case, the pair $(\mathcal{X}, \preceq)$ is said to be a poset.

Let $\preceq$ be a partial order on $\mathcal{X}$.

A set $U \subset \mathcal{X}$ is said to be an upper set when for any $x \in U$ and any $y \in \mathcal{X}$ such that $x \preceq y$, it holds that $y \in U$.

An upper quadrant set is a subset of $\mathcal{X}$ of the form $Q_{x}=\{y \in \mathcal{X} \mid x \preceq y\}$, with $x \in \mathcal{X}$.

A mapping $f: \mathcal{X} \rightarrow \mathbb{R}$ is said to be $\preceq$-preserving if given $x, y \in \mathcal{X}$ with $x \preceq y$, then $f(x) \leq f(y)$.

A partial order $\preceq$ on $\mathbb{R}^{n}$ is said to be closed when given $\left\{x_{m}\right\}_{m}$ and $\left\{y_{m}\right\}_{m}$ subsets of $\mathbb{R}^{n}$ with $x_{m} \preceq y_{m}$, and $x, y \in \mathbb{R}^{n}$ such that $\lim _{m} x_{m}=x$ and $\lim _{m} y_{m}=y$, then $x \preceq y$. 
The reader is referred, for instance, to Neggers and Kim (1998) and Schröder (2003) for an introduction to the theory of ordered sets.

Stochastic orders try to order probabilities in accordance with an appropriate criterion. Formally a stochastic order can be defined as a pre-order on a set of probabilities associated with a measurable space. The reader is refereed to the books Müller and Stoyan (2002), Shaked and Shanthikumar (2007) and Belzunce et al. (2016) for a detailed and meticulous introduction to stochastic orders from both theoretical and applied points of view.

Given a random vector $\mathbf{X}, F_{\mathbf{X}}$ will denote its distribution function, $\bar{F}_{\mathbf{X}}$ will stand for its survival function and $E \mathbf{X}$ will be its expected value.

Some stochastic orders which will appear in the paper are the following. Let $\mathbf{X}=\left(X_{1}, \ldots, X_{n}\right)$ and $\mathbf{Y}=\left(Y_{1}, \ldots, Y_{n}\right)$ be random vectors,

i) $\mathbf{X}$ is said to be smaller than $\mathbf{Y}$ in the usual stochastic order, denoted by $\mathbf{X} \preceq_{s t} \mathbf{Y}$, if $E f(\mathbf{X}) \leq E f(\mathbf{Y})$ for all increasing functions $f: \mathbb{R}^{n} \rightarrow \mathbb{R}$ for which the expectations exist,

ii) $\mathbf{X}$ is said to be smaller than $\mathbf{Y}$ in the upper orthant order, denoted by $\mathbf{X} \preceq_{u o} \mathbf{Y}$, if $\bar{F}_{\mathbf{X}}(z) \leq \bar{F}_{\mathbf{Y}}(z)$ for all $z \in \mathbb{R}^{n}$,

iii) $\mathbf{X}$ is said to be smaller than $\mathbf{Y}$ in the lower orthant order, denoted by $\mathbf{X} \preceq_{l o} \mathbf{Y}$, if $F_{\mathbf{X}}(z) \geq F_{\mathbf{Y}}(z)$ for all $z \in \mathbb{R}^{n}$.

Some orders to compare random cash flows are introduced in Scarsini (1986). We include here their definitions for random vectors. Let $\mathbf{X}=$ $\left(X_{1}, \ldots, X_{n}\right)$ and $\mathbf{Y}=\left(Y_{1}, \ldots, Y_{n}\right)$ be random vectors,

$i v) \mathbf{X}$ is said to be smaller than $\mathbf{Y}$ in the order $\preceq^{1}$, denoted by $\mathbf{X} \preceq^{1} \mathbf{Y}$, if $\phi_{1} X_{1}+\ldots+\phi_{n} X_{n} \preceq_{s t} \phi_{1} Y_{1}+\ldots+\phi_{n} Y_{n}$ for all $\phi=\left(\phi_{1}, \phi_{2}, \ldots, \phi_{n}\right)$ with $1 \geq \phi_{1} \geq \phi_{2} \ldots \geq \phi_{n} \geq 0$

v) $\mathbf{X}$ is said to be smaller than $\mathbf{Y}$ in the order $\preceq^{2}$, denoted by $\mathbf{X} \preceq^{2} \mathbf{Y}$, if $E\left(\phi_{1} v\left(X_{1}\right)+\ldots+\phi_{n} v\left(X_{n}\right)\right) \leq E\left(\phi_{1} v\left(Y_{1}\right)+\ldots+\phi_{n} v\left(Y_{n}\right)\right)$ for all $\phi=$ $\left(\phi_{1}, \phi_{2}, \ldots, \phi_{n}\right)$ with $1 \geq \phi_{1} \geq \phi_{2} \ldots \geq \phi_{n} \geq 0$ and all increasing function $v: \mathbb{R} \rightarrow \mathbb{R}$.

A stochastic order $\preceq$ is said to be integral when there exists a set $\mathcal{F}$ of measurable real mappings such that two probabilities $P_{1}$ and $P_{2}$ satisfy

$$
P_{1} \preceq P_{2} \quad \text { when } \quad \int f d P_{1} \leq \int f d P_{2}
$$

for any $f \in \mathcal{F}$ such that the above integrals exist. The set of mappings $\mathcal{F}$ is said to be a generator of the order.

An important generator is the so-called maximal generator. Roughly 
speaking, the maximal generator is the largest generator of an integral stochastic order inside an appropriate class of mappings.

The concept of maximal generator of an integral stochastic order is associated with the so-called weight function, a measurable mapping $b: \mathbb{R}^{n} \rightarrow$ $[1, \infty)$.

The $b$-norm of a mapping $f: \mathbb{R}^{n} \rightarrow \mathbb{R}$ is $\|f\|_{b}=\sup _{x \in \mathbb{R}^{n}} \frac{|f(x)|}{b(x)}$.

The set of $b$-bounded measurable mappings will be denoted by $\mathcal{M}_{b}$. We will denote by $\mathcal{P}_{b}$ the class of probabilities $P$ on $\left(\mathbb{R}^{n}, \mathcal{B}_{n}\right)$ such that

$$
\int_{\mathbb{R}^{n}} b d P<\infty
$$

Note that $\int_{\mathbb{R}^{n}} f d P \in \mathbb{R}$ for any $f \in \mathcal{M}_{b}$ and $P \in \mathcal{P}_{b}$.

The maximal generator for a weight function $b$ of an integral stochastic order $\preceq$ on $\mathcal{P}_{b}$, is the set of all mappings $f \in \mathcal{M}_{b}$ such that

$$
P \preceq Q \quad \text { implies } \quad \int_{\mathbb{R}^{n}} f d P \leq \int_{\mathbb{R}^{n}} f d Q .
$$

The analysis of the maximal generator is quite important for the study of an integral stochastic order, since many properties of the order can be easily deduced by means of simple conditions of the mappings in the maximal generator.

The reader is referred to Müller (1997) and Müller and Stoyan (2002) (Chapter 2) for a rigorous analysis of integral stochastic orders.

Let $P$ be a probability on $\left(\mathbb{R}^{n}, \mathcal{B}_{n}\right)$ and let $T: \mathbb{R}^{n} \rightarrow \mathbb{R}^{n}$ be a measurable mapping. Then $P \circ T^{-1}$ will denote the probability on $\mathcal{B}_{n}$ given by $P \circ$ $T^{-1}(B)=P\left(T^{-1}(B)\right)$ for any $B \in \mathcal{B}_{n}$.

We will denote with the symbol $\sim_{s t}$ the equality in distribution.

Throughout the paper $\leq$ will stand for the usual componentwise order on $\mathbb{R}^{n}$.

\section{The new stochastic order: main results}

In this section we define the new stochastic order motivated by the time value of money. Afterwards we study in detail such an order.

We introduce the following class of mappings to define the new order. 
Let $e_{i} \in \mathbb{R}^{n}$ be the $i^{\text {th }}$-unit vector, with $1 \leq i \leq n$. Let $\mathcal{F}=\left\{f: \mathbb{R}^{n} \rightarrow \mathbb{R} \mid\right.$

i) $f\left(x+\varepsilon_{i} e_{i}\right) \geq f\left(x+\varepsilon_{i+1} e_{i+1}\right)$ for all $x \in \mathbb{R}^{n}, 0 \leq \varepsilon_{i+1} \leq \varepsilon_{i}, 1 \leq i \leq n-1$,

ii) $f\left(x+\varepsilon_{n} e_{n}\right) \geq f(x)$ for all $x \in \mathbb{R}^{n}$ and $\left.0 \leq \varepsilon_{n}\right\}$.

These mappings capture the basic fact that an amount of money in year $i$ is preferable to a non-greater amount in year $i+1$. Clearly those mappings are increasing.

Definition 3.1. Let $\boldsymbol{X}=\left(X_{1}, \ldots, X_{n}\right)$ and $\boldsymbol{Y}=\left(Y_{1}, \ldots, Y_{n}\right)$ be random vectors. It will be said that $\boldsymbol{X}$ is less than $\boldsymbol{Y}$ in the time value of money stochastic order if $E(f(\boldsymbol{X})) \leq E(f(\boldsymbol{Y}))$ for any $f \in \mathcal{F}$ such that the above expectations exist. This relation will be denoted by $\boldsymbol{X} \preceq_{t v m} \boldsymbol{Y}$.

If $\mathbf{X}=\left(X_{1}, \ldots, X_{n}\right)$ and $\mathbf{Y}=\left(Y_{1}, \ldots, Y_{n}\right)$ are random vectors whose $i^{\text {th }}$ components represent the annual cash flows at the end of the $i^{\text {th }}$ year in $n$-year investments, the relation $\mathbf{X} \preceq_{\text {tvm }} \mathbf{Y}$ means that the expected profitability of the investment associated with the random vector $\mathbf{Y}$, is at least as high as the expected profitability of the investment associated with $\mathbf{X}$, whatever mapping $f \in \mathcal{F}$ represents the final profitability of the investment.

\subsection{Characterizations of the stochastic order $\preceq_{t v m}$}

In this subsection we develop different key characterizations of the new stochastic order. By means of those characterization results, important properties of the order will be derived. In particular, we obtain the maximal generator of the time value of money stochastic order.

We take the weight function $b=1$ including all the probabilities on $\left(\mathbb{R}^{n}, \mathcal{B}_{n}\right)$ in the class $\mathcal{P}_{b}$. Thus the $b$-norm is the supremum norm and $\mathcal{M}_{b}$ is the class of measurable and bounded mappings.

Now we provide an alternative representation of the class of mappings $\mathcal{F}$.

Let $\widetilde{e}_{i}=e_{i}-e_{i+1}$, with $1 \leq i \leq n-1$, and $\widetilde{e}_{n}=e_{n}$. We define the class of mappings

$$
\widetilde{\mathcal{F}}=\left\{f: \mathbb{R}^{n} \rightarrow \mathbb{R} \mid f\left(x+\varepsilon \widetilde{e}_{i}\right) \geq f(x) \text { for all } x \in \mathbb{R}^{n}, 0 \leq \varepsilon, 1 \leq i \leq n\right\} .
$$

The following technical result will be key to derive the maximal generator of the new order.

Let $\mathcal{F}_{b}$ and $\widetilde{\mathcal{F}}_{b}$ be the classes of bounded mappings in $\mathcal{F}$ and $\widetilde{\mathcal{F}}$ respectively. 
Lemma 3.2. It holds that $\mathcal{F}=\widetilde{\mathcal{F}}$ and $\widetilde{\mathcal{F}}_{b}=\mathcal{F}_{b}$.

Proof. Let $f \in \mathcal{F}$. Given $x \in \mathbb{R}^{n}, \varepsilon \geq 0$, and $1 \leq i \leq n-1$, we have that $f\left(x+\varepsilon \widetilde{e}_{i}\right)=f\left(x+\varepsilon e_{i}-\varepsilon e_{i+1}\right) \geq f\left(x+\varepsilon e_{i+1}-\varepsilon e_{i+1}\right)=f(x)$ since $f \in \mathcal{F}$. Clearly $f\left(x+\varepsilon \widetilde{e}_{n}\right) \geq f(x)$. Therefore $\mathcal{F} \subset \widetilde{\mathcal{F}}$.

Conversely, let $f \in \widetilde{\mathcal{F}}$.

First of all, note that given $x \in \mathbb{R}^{n}$ and $\varepsilon \geq 0$, it holds that $f\left(x+\varepsilon e_{i}\right)=$ $f\left(x+\varepsilon \widetilde{e}_{i}+\varepsilon \widetilde{e}_{i+1}+\cdots+\varepsilon \widetilde{e}_{n}\right) \geq f\left(x+\varepsilon \widetilde{e}_{i}+\varepsilon \widetilde{e}_{i+1}+\cdots+\varepsilon \widetilde{e}_{n-1}\right) \geq f\left(x+\varepsilon \widetilde{e}_{i}+\right.$ $\left.\varepsilon \widetilde{e}_{i+1}+\cdots+\varepsilon \widetilde{e}_{n-2}\right) \geq \ldots \geq f\left(x+\varepsilon \widetilde{e}_{i}\right) \geq f(x)$. That is, any mapping of $\widetilde{\mathcal{F}}$ is increasing.

Now let $x \in \mathbb{R}^{n}, 1 \leq i \leq n-1,0 \leq \varepsilon_{i+1} \leq \varepsilon_{i}$. We have that $f\left(x+\varepsilon_{i} e_{i}\right)=$ $f\left(x+\varepsilon_{i} \widetilde{e}_{i}+\varepsilon_{i} e_{i+1}\right) \geq f\left(x+\varepsilon_{i} e_{i+1}\right)$ since $f \in \widetilde{\mathcal{F}}$. Note that $f$ is increasing, and so $f\left(x+\varepsilon_{i} e_{i+1}\right) \geq f\left(x+\varepsilon_{i+1} e_{i+1}\right)$. On the other hand, $f\left(x+\varepsilon_{n} e_{n}\right)=$ $f\left(x+\varepsilon_{n} \widetilde{e}_{n}\right) \geq f(x)$ for any $x \in \mathbb{R}^{n}$ and $\varepsilon_{n} \geq 0$. Thus $\widetilde{\mathcal{F}} \subset \mathcal{F}$.

Since $\widetilde{\mathcal{F}}=\mathcal{F}$, we obtain that $\widetilde{\mathcal{F}}_{b}=\mathcal{F}_{b}$

Let $h: \mathbb{R}^{n} \rightarrow \mathbb{R}^{n}$ be the linear map such that $h\left(e_{i}\right)=\widetilde{e_{i}}$ for all $1 \leq i \leq n$. Note that $h$ is a linear bijection.

The following result provides a key characterization of the new order.

Theorem 3.3. Let $\boldsymbol{X}=\left(X_{1}, \ldots, X_{n}\right)$ and $\boldsymbol{Y}=\left(Y_{1}, \ldots, Y_{n}\right)$ be random vectors. Then $\boldsymbol{X} \preceq_{\text {tvm }} \boldsymbol{Y}$ if and only if $h^{-1}(\boldsymbol{X}) \preceq_{s t} h^{-1}(\boldsymbol{Y})$.

Proof. Let $\mathcal{I}=\left\{f: \mathbb{R}^{n} \rightarrow \mathbb{R} \mid f\right.$ is increasing $\}$. Recall that $\mathcal{I}$ is a generator of the usual stochastic order. Consider $\mathcal{I}_{h^{-1}}=\left\{f \circ h^{-1} \mid f \in \mathcal{I}\right\}$.

Let us see that the class of mappings $\widetilde{\mathcal{F}}$ and the class $\mathcal{I}_{h^{-1}}$ are equal.

Let $f \in \widetilde{\mathcal{F}}$. We have that $f \in \mathcal{I}_{h^{-1}}$ when $f \circ h \in \mathcal{I}$, that is, $f \circ h$ is increasing. Then, for all $x \in \mathbb{R}^{n}, \varepsilon \geq 0$ and $1 \leq i \leq n$, by the definition of $h$, $f \circ h\left(x+\varepsilon e_{i}\right)=f\left(h(x)+\varepsilon h\left(e_{i}\right)\right)=f\left(h(x)+\varepsilon \widetilde{e}_{i}\right) \geq f(h(x))=f \circ h(x)$ since $f \in \widetilde{\mathcal{F}}$. As a consequence, $f \circ h$ is increasing, and so $f \in \mathcal{I}_{h^{-1}}$. Therefore, $\widetilde{\mathcal{F}} \subset \mathcal{I}_{h^{-1}}$.

Now, let $g \in \mathcal{I}_{h^{-1}}$, that is, $g=f \circ h^{-1}$ with $f \in \mathcal{I}$.

For all $x \in \mathbb{R}^{n}, \varepsilon \geq 0$ and $1 \leq i \leq n$, by the definition of $h$, we have that $g\left(x+\varepsilon \widetilde{e}_{i}\right)=f \circ h^{-1}\left(x+\varepsilon \widetilde{e}_{i}\right)=f\left(h^{-1}(x)+\varepsilon h^{-1}\left(\widetilde{e}_{i}\right)\right)=f\left(h^{-1}(x)+\varepsilon e_{i}\right) \geq$ $f\left(h^{-1}(x)\right)=f \circ h^{-1}(x)=g(x)$ since $f \in \mathcal{I}$, that is, $f$ is increasing. Thus, $g \in \widetilde{\mathcal{F}}$, and so $\mathcal{I}_{h^{-1}} \subset \widetilde{\mathcal{F}}$. 
Now, we have that $\mathcal{I}_{h^{-1}}=\widetilde{\mathcal{F}}$, and, as a consequence, $\mathcal{I}_{h^{-1}}$ is a generator of the stochastic order $\preceq_{t v m}$. Then, $\mathbf{X} \preceq_{t v m} \mathbf{Y}$ is equivalent to

$$
\int_{\mathbb{R}^{n}} f \circ h^{-1} d P_{\mathbf{X}} \leq \int_{\mathbb{R}^{n}} f \circ h^{-1} d P_{\mathbf{Y}},
$$

for all $f \in \mathcal{I}$ such that the integrals exist. By a change of variable (see, for instance, Halmos (1950), page 163) the above inequality is the same as

$$
\int_{\mathbb{R}^{n}} f d P_{\mathbf{X}} \circ h \leq \int_{\mathbb{R}^{n}} f d P_{\mathbf{Y}} \circ h
$$

for all $f \in \mathcal{I}$ such that the integrals exist.

Note that for any $B \in \mathcal{B}_{n}, P_{\mathbf{X}} \circ h(B)=P_{h^{-1}(\mathbf{X})}(B)$, and so we have that $\mathbf{X} \preceq_{\text {tvm }} \mathbf{Y}$ is equivalent to

$$
\int_{\mathbb{R}^{n}} f d P_{h^{-1}(\mathbf{X})} \leq \int_{\mathbb{R}^{n}} f d P_{h^{-1}(\mathbf{Y})},
$$

for all $f \in \mathcal{I}$ such that the integrals exist. Then, $\mathbf{X} \preceq_{t v m} \mathbf{Y}$ if and only if $h^{-1}(\mathbf{X}) \preceq_{s t} h^{-1}(\mathbf{Y})$, since $\mathcal{I}$, the class of increasing mappings from $\mathbb{R}^{n}$ to $\mathbb{R}$, is a generator of the usual multivariate stochastic order.

Corollary 3.4. The stochastic order $\preceq_{t v m}$ is a partial order.

Proof. Reflexivity and transitivity are clear. Now, suppose two random vectors $\mathbf{X}$ and $\mathbf{Y}$ satisfying that $\mathbf{X} \preceq_{t v m} \mathbf{Y}$ and $\mathbf{Y} \preceq_{t v m} \mathbf{X}$. By Theorem 3.3, we obtain that $h^{-1}(\mathbf{X}) \sim_{s t} h^{-1}(\mathbf{Y})$. Since $h$ is bijective, $\mathbf{X} \sim_{s t} \mathbf{Y}$.

The following theorem provides the maximal generator of the time value of money stochastic order for the weight function $b=1$.

Theorem 3.5. The class $\mathcal{F}_{b}$ is the maximal generator of $\preceq_{\text {tvm }}$.

Proof. By Lemma 3.2, we have that $\mathcal{F}_{b}=\widetilde{\mathcal{F}}_{b}$. We will prove that $\widetilde{\mathcal{F}}_{b}$ is a generator of $\preceq_{t v m}$ which is a convex cone, containing the constant functions, and being closed under pointwise convergence inside $\mathcal{M}_{b}$.

The proof of Theorem 3.3 provides that $\widetilde{\mathcal{F}}_{b}$ is equal to $\left(\mathcal{I}_{b}\right)_{h^{-1}}=\left\{f \circ h^{-1} \mid\right.$ $\left.f \in \mathcal{I}_{b}\right\}$ where $\mathcal{I}_{b}=\left\{f: \mathbb{R}^{n} \rightarrow \mathbb{R} \mid f\right.$ is bounded and increasing $\}$ is the maximal generator of $\preceq_{s t}$. Then $\widetilde{\mathcal{F}}_{b}$ is a generator of $\preceq_{t v m}$ because of Theorem 3.3 .

It is clear that $\widetilde{\mathcal{F}}_{b}$ is a convex cone with the constant mappings. 
Let $g: \mathbb{R}^{n} \rightarrow \mathbb{R}$ be a bounded measurable mapping such that there exits a sequence $\left\{g_{m}\right\}_{m} \subset \widetilde{\mathcal{F}}_{b}$ with $\lim _{m} g_{m}(x)=g(x)$ for all $x \in \mathbb{R}^{n}$. We have that $g_{m}$ belongs to $\left(\mathcal{I}_{b}\right)_{h^{-1}}$ for any $m \in \mathbb{N}$, and so $g_{m}=f_{m} \circ h^{-1}$ where $f_{m}$ belongs to $\mathcal{I}_{b}$.

Now, $\lim _{m} g_{m}(x)=\lim _{m} f_{m}\left(h^{-1}(x)\right)=g(x)=g \circ h\left(h^{-1}(x)\right)$. But $\mathcal{I}_{b}$ is closed under pointwise convergence inside of $\mathcal{M}_{b}$, and then $g \circ h$ is in $\mathcal{I}_{b}$. As a consequence, $g$ belongs to $\left(\mathcal{I}_{b}\right)_{h^{-1}}=\widetilde{\mathcal{F}}_{b}=\mathcal{F}_{b}$.

Now the result is a consequence of Corollary 2.3.9 in Müller and Stoyan (2002).

We relate the stochastic order $\preceq_{t v m}$ with stochastic orders generated by means of a partial order on $\mathbb{R}^{n}$ and the class of preserving mappings of such an order. Those orders have been widely studied in mathematical literature. The reader is referred for instance to Strassen (1965), Kamae et al. (1977), Kamae and Krengel (1978), Müller and Stoyan (2002) (Section 2.6), LópezDíaz and López-Díaz (2012), López-Díaz and López-Díaz (2013b) and their references for this kind of stochastic orders, and to López-Díaz and LópezDíaz (2013a) for an application of those orders to economics.

A partial order $\preceq$ on $\mathbb{R}^{n}$ generates a stochastic order on the class of probabilities associated with the usual Borel measurable space, let us denote such an order by $\preceq^{g}$, as follows

$$
\mathbf{X} \preceq^{g} \mathbf{Y} \quad \text { when } \quad \int_{\mathbb{R}^{n}} f d P_{\mathbf{X}} \leq \int_{\mathbb{R}^{n}} f d P_{\mathbf{Y}}
$$

for any bounded and measurable $\preceq$-preserving mapping $f: \mathbb{R}^{n} \rightarrow \mathbb{R}$.

We will prove that the stochastic order $\preceq_{t v m}$ is generated by the partial order $\leq_{h^{-1}}$ on $\mathbb{R}^{n}$ given by $x \leq_{h^{-1}} y$ when $h^{-1}(x) \leq h^{-1}(y)$, where $\leq$ stands for the usual componentwise order. Note that $\left(\mathbb{R}^{n}, \leq_{h^{-1}}\right)$ is a poset because $h^{-1}$ is a linear bijection. This will lead to important characterizations of the new order.

Theorem 3.6. The stochastic orders $\leq_{h^{-1}}^{g}$ and $\preceq_{\text {tvm }}$ are the same.

Proof. Let $\mathcal{F}_{b}^{\leq_{h^{-1}}}$ be the class of bounded and measurable $\leq_{h^{-1} \text {-preserving }}$ mappings.

Let us see that $\mathcal{F}_{b}^{\leq_{h^{-1}}}$ is equal to $\left(\mathcal{I}_{b}\right)_{h^{-1}}$. Note that $h$ is continuous, and thus measurable.

Let $f \in \mathcal{F}_{b}^{\leq{ }^{-1}}$. Thus if $h^{-1}(x) \leq h^{-1}(y)$, we have that $f(x) \leq f(y)$. 
Let $x, y \in \mathbb{R}^{n}$ with $x \leq y$. Then $h^{-1}(h(x)) \leq h^{-1}(h(y))$, which implies that $f(h(x)) \leq f(h(y))$. Hence $f \circ h$ is increasing and bounded, and so $f \in\left(\mathcal{I}_{b}\right)_{h^{-1}}$. Thus $\mathcal{F}_{b}^{\leq_{h^{-1}}} \subset\left(\mathcal{I}_{b}\right)_{h^{-1}}$.

Conversely, if $f \in\left(\mathcal{I}_{b}\right)_{h^{-1}}$, then $f=g \circ h^{-1}$ with $g$ a bounded and increasing map. Let $x, y \in \mathbb{R}^{n}$ with $x \leq_{h^{-1}} y$, then $h^{-1}(x) \leq h^{-1}(y)$ and so $g \circ h^{-1}(x) \leq g \circ h^{-1}(y)$, that is, $f$ is $\leq_{h^{-1}}$-preserving.

As a consequence $\mathcal{F}_{b}^{\leq h^{-1}}$ and $\left(\mathcal{I}_{b}\right)_{h^{-1}}$ are the same class of mappings, and $\widetilde{\mathcal{F}}_{b}=\left(\mathcal{I}_{b}\right)_{h^{-1}}$, which derives the result.

Lemma 3.7. The partial order $\leq_{h^{-1}}$ is closed.

Proof. Let $\left\{x_{m}\right\}_{m}$ and $\left\{y_{m}\right\}_{m}$ be subsets of $\mathbb{R}^{n}$ with $x_{m} \leq_{h^{-1}} y_{m}$, and $x, y \in$ $\mathbb{R}^{n}$ such that $\lim _{m} x_{m}=x$ and $\lim _{m} y_{m}=y$.

We have that $x_{m} \leq_{h^{-1}} y_{m}$ means $h^{-1}\left(x_{m}\right) \leq h^{-1}\left(y_{m}\right)$. Since $h^{-1}$ is continuous, we obtain that $h^{-1}(x) \leq h^{-1}(y)$, and so $x \leq_{h^{-1}} y$.

Thus $\preceq_{t v m}$ is a stochastic order generated by means of the closed partial order $\leq_{h^{-1}}$ and the class of bounded and measurable $\leq_{h^{-1}}$-preserving mappings. This leads to other important characterizations of $\preceq_{t v m}$.

Proposition 3.8. Let $\boldsymbol{X}=\left(X_{1}, \ldots, X_{n}\right)$ and $\boldsymbol{Y}=\left(Y_{1}, \ldots, Y_{n}\right)$ be random vectors. The following conditions are equivalent:

i) $\boldsymbol{X} \preceq_{\text {tvm }} \boldsymbol{Y}$,

ii) there exists a probability space $(\Omega, \mathcal{A}, \operatorname{Pr})$ and $\widehat{\boldsymbol{X}}, \widehat{\boldsymbol{Y}}: \Omega \rightarrow \mathbb{R}^{n}$ random vectors, inducing the probabilities $P_{\boldsymbol{X}}$ and $P_{\boldsymbol{Y}}$ respectively, such that $\widehat{\boldsymbol{X}} \leq_{h^{-1}}$ $\widehat{\boldsymbol{Y}}$ a.s. $[\operatorname{Pr}]$,

iii) $P_{\boldsymbol{X}}(U) \leq P_{\boldsymbol{Y}}(U)$ for any measurable upper set $U$ with respect to the partial order $\leq_{h^{-1}}$,

iv) $P_{\boldsymbol{X}}(U) \leq P_{\boldsymbol{Y}}(U)$ for any closed measurable upper set $U$ with respect to the partial order $\leq_{h^{-1}}$,

v) $\int_{\mathbb{R}^{n}} f d P_{\boldsymbol{X}} \leq \int_{\mathbb{R}^{n}} f d P_{\boldsymbol{Y}}$ for all bounded, continuous and $\leq_{h^{-1}}$-preserving mappings $f$.

Proof. Since the partial order $\leq_{h^{-1}}$ is closed, the result follows from Strassen (1965) and Kamae et al. (1977). See also Theorem 2.6.3 and 2.6.4 in Müller and Stoyan (2002). 


\subsection{Properties of the stochastic order $\preceq_{t v m}$}

Main properties of the new stochastic order are studied in this subsection.

Proposition 3.9. The stochastic order $\preceq_{\text {tvm }}$ is closed under convolutions.

Proof. Let $f \in \mathcal{F}_{b}$ and $a \in \mathbb{R}^{n}$. We define the mapping $f_{a}: \mathbb{R}^{n} \rightarrow \mathbb{R}$ with $f_{a}(x)=f(a+x)$. The map $f_{a}$ is bounded and measurable. Moreover, given $1 \leq i \leq n-1,0 \leq \varepsilon_{i+1} \leq \varepsilon_{i}$, and $x \in \mathbb{R}^{n}$, we have that $f_{a}\left(x+\varepsilon_{i} e_{i}\right)=$ $f\left(a+x+\varepsilon_{i} e_{i}\right) \geq f\left(a+x+\varepsilon_{i+1} e_{i+1}\right)=f_{a}\left(x+\varepsilon_{i+1} e_{i+1}\right)$. In the same way, if $\varepsilon_{n} \geq 0$ we have that $f_{a}\left(x+\varepsilon_{n} e_{n}\right)=f\left(a+x+\varepsilon_{n} e_{n}\right) \geq f(a+x) \geq f_{a}(x)$.

Therefore $f_{a} \in \mathcal{F}_{b}$ for any $a \in \mathbb{R}^{n}$, which implies the result in accordance with Theorem 2.4.2 in Müller and Stoyan (2002).

The new order is closed with respect to independent concatenation.

Proposition 3.10. Let $\boldsymbol{X}_{1}, \boldsymbol{X}_{2}, \boldsymbol{Y}_{1}$ and $\boldsymbol{Y}_{2}$ be independent random vectors such that $\boldsymbol{X}_{1} \preceq_{\text {tvm }} \boldsymbol{Y}_{1}$ and $\boldsymbol{X}_{2} \preceq_{\text {tvm }} \boldsymbol{Y}_{2}$. Then $\left(\boldsymbol{X}_{1}, \boldsymbol{X}_{2}\right) \preceq_{\text {tvm }}\left(\boldsymbol{Y}_{1}, \boldsymbol{Y}_{2}\right)$.

Proof. Let us suppose that $\mathbf{X}_{i}$ and $\mathbf{Y}_{i}$ have dimension $n_{i}, 1 \leq i \leq 2$. Let $\mathcal{F}_{b}^{n_{i}}$ denote the class of mappings defined on $\mathbb{R}^{n_{i}}$ with the same conditions as the ones in $\mathcal{F}_{b}$.

Let $f \in \mathcal{F}_{b}^{n_{1}+n_{2}}$. The result is proved if we see that $f(x, \cdot): \mathbb{R}^{n_{2}} \rightarrow \mathbb{R}$ belongs to $\mathcal{F}_{b}^{n_{2}}$ for any $x \in \mathbb{R}^{n_{1}}$, and $f(\cdot, y): \mathbb{R}^{n_{1}} \rightarrow \mathbb{R}$ belongs to $\mathcal{F}_{b}^{n_{1}}$ for any $y \in \mathbb{R}^{n_{2}}$ (see Theorem 3.2.3 in Müller and Stoyan (2002)).

Let $x \in \mathbb{R}^{n_{1}}$, the mapping $f(x, \cdot)$ is bounded and measurable. On the other hand, $f\left(x, z+\varepsilon_{i} e_{i}\right) \geq f\left(x, z+\varepsilon_{i+1} e_{i+1}\right)$ for any $z \in \mathbb{R}^{n_{2}}$ and $0 \leq \varepsilon_{i+1} \leq$ $\varepsilon_{i}$ with $1 \leq i \leq n_{2}-1$, since $\left(x, z+\varepsilon_{i} e_{i}\right)=(x, z)+\varepsilon_{i} e_{n_{1}+i},\left(x, z+\varepsilon_{i+1} e_{i+1}\right)=$ $(x, z)+\varepsilon_{i+1} e_{n_{1}+i+1}$ and $f \in \mathcal{F}_{b}^{n_{1}+n_{2}}$. In a similar way it is possible to prove that $f\left(x, z+\varepsilon_{n} e_{n}\right) \geq f(x, z)$ for all $z \in \mathbb{R}^{n_{2}}$ and $0 \leq \varepsilon_{n}$.

Therefore $f(x, \cdot) \in \mathcal{F}_{b}^{n_{2}}$ for any $x \in \mathbb{R}^{n_{1}}$.

With the same procedure, we obtain that $f(\cdot, y) \in \mathcal{F}_{b}^{n_{1}}$ for any $y \in \mathbb{R}^{n_{2}}$, which proves the proposition.

Proposition 3.11. The stochastic order $\preceq_{\text {tvm }}$ is closed under the weak convergence.

Proof. By Proposition $3.8 v$ ), there exists a generator of the order with bounded and continuous mappings, which implies the result.

Proposition 3.12. The order $\preceq_{t v m}$ is closed under mixture. 
Proof. Note that the order $\preceq_{t v m}$ is integral.

Proposition 3.13. Let $\boldsymbol{X}=\left(X_{1}, \ldots, X_{n}\right)$ and $\boldsymbol{Y}=\left(Y_{1}, \ldots, Y_{n}\right)$ be random vectors such that $\boldsymbol{X} \preceq_{t v m} \boldsymbol{Y}$. Then,

i) $\alpha \boldsymbol{X} \preceq$ tvm $\alpha \boldsymbol{Y}$ for all $\alpha \in[0, \infty)$,

ii) $\boldsymbol{X} \preceq_{\text {tvm }} \boldsymbol{X}+a$ for all $a \in \mathbb{R}^{n}$ with $a \geq 0$.

Proof. $i)$ The case $\alpha=0$ is trivial. Let $\alpha>0$. In accordance with Theorem 3.3, $\mathbf{X} \preceq_{t v m} \mathbf{Y}$ is equivalent to $h^{-1}(\mathbf{X}) \preceq_{s t} h^{-1}(\mathbf{Y})$, which implies that $\alpha h^{-1}(\mathbf{X}) \preceq_{s t} \alpha h^{-1}(\mathbf{Y})$. That is the same as $h^{-1}(\alpha \mathbf{X}) \preceq_{s t} h^{-1}(\alpha \mathbf{Y})$. Now Theorem 3.3 ensures that $\alpha \mathbf{X} \preceq_{t v m} \alpha \mathbf{Y}$.

ii) Observe that the mappings in $\mathcal{F}_{b}$ are increasing.

Let $a=\left(a_{1}, a_{2}, \ldots, a_{n}\right) \in \mathbb{R}^{n}$, we will denote by $a_{(1)}, a_{(2)}, \ldots, a_{(n)}$ the components of $a$ in increasing order, that is, $a_{(1)} \leq a_{(2)} \leq \ldots \leq a_{(n)}$, and by $a_{[1]}, a_{[2]}, \ldots, a_{[n]}$ the components of $a$ in decreasing order, so $a_{[1]} \geq a_{[2]} \geq$ $\ldots \geq a_{[n]}$.

By $a^{\uparrow}$ we will denote the vector $\left(a_{(1)}, a_{(2)}, \ldots, a_{(n)}\right)$, and $a^{\downarrow}$ will stand for the rearrangement $\left(a_{[1]}, a_{[2]}, \ldots, a_{[n]}\right)$.

Now note that $x \leq_{h^{-1}} y$ when $h^{-1}(x) \leq h^{-1}(y)$. Thus $x \leq_{h^{-1}} y$ when $\sum_{i=1}^{k} x_{i} \leq \sum_{i=1}^{k} y_{i}$ for any $1 \leq k \leq n$.

The following result relates the new order with the increasing and decreasing rearrangements of the components of random vectors.

Proposition 3.14. Let $\boldsymbol{X}=\left(X_{1}, \ldots, X_{n}\right)$ be a random vector. Then $\boldsymbol{X}^{\uparrow} \preceq_{t v m}$ $\boldsymbol{X} \preceq_{t v m} \boldsymbol{X}^{\downarrow}$.

Proof. Given any $x \in \mathbb{R}^{n}$ we have that $\sum_{i=1}^{k} x_{i} \leq \sum_{i=1}^{k} x_{[i]}$ for any $1 \leq k \leq n$. Then $x \leq_{h^{-1}} x^{\downarrow}$. Thus $f(x) \leq f\left(x^{\downarrow}\right)$ for any $f \leq_{h^{-1}}$-preserving mapping. As a consequence, $f(\mathbf{X}) \leq f\left(\mathbf{X}^{\downarrow}\right)$, which implies that $\mathbf{X} \preceq_{t v m} \mathbf{X}^{\downarrow}$ in accordance with $v$ ) in Proposition 3.8.

In a similar way we obtain that $\sum_{i=1}^{k} x_{(i)} \leq \sum_{i=1}^{k} x_{i}$ for any $x \in \mathbb{R}^{n}$ and $1 \leq k \leq n$. Thus $x^{\uparrow} \leq_{h^{-1}} x$, which implies that $f\left(\mathbf{X}^{\uparrow}\right) \leq f(\mathbf{X})$ for any $f \leq_{h^{-1}}$-preserving mapping, and so $\mathbf{X}^{\uparrow} \preceq_{t v m} \mathbf{X}$ applying $v$ ) in Proposition 3.8 .

The time value of money stochastic order is preserved by the marginalization of the first $k$ components with $1 \leq k \leq n$. 
Proposition 3.15. Let $\boldsymbol{X}=\left(X_{1}, \ldots, X_{n}\right)$ and $\boldsymbol{Y}=\left(Y_{1}, \ldots, Y_{n}\right)$ be random vectors such that $\boldsymbol{X} \preceq_{\text {tvm }} \boldsymbol{Y}$. Then it holds that $\left(X_{1}, \ldots, X_{k}\right) \preceq_{\text {tvm }}$ $\left(Y_{1}, \ldots, Y_{k}\right)$ for any $1 \leq k \leq n$.

Proof. The condition $\mathbf{X} \preceq_{t v m} \mathbf{Y}$ is equivalent to $h^{-1}(\mathbf{X}) \preceq_{s t} h^{-1}(\mathbf{Y})$ by Theorem 3.3. Since the usual multivariate stochastic order is closed under marginalization, $\left(h^{-1}(X)_{1}, \ldots, h^{-1}(X)_{k}\right) \preceq_{s t}\left(h^{-1}(Y)_{1}, \ldots, h^{-1}(Y)_{k}\right)$. Now $\left(h^{-1}(X)_{1}, \ldots, h^{-1}(X)_{k}\right)=h_{\left.\right|_{k}}^{-1}\left(\left(X_{1}, \ldots, X_{k}\right)\right)$ where $h_{\left.\right|_{k}}: \mathbb{R}^{k} \rightarrow \mathbb{R}^{k}$ is defined in the same way as $h$ from $\mathbb{R}^{n}$ to $\mathbb{R}^{n}$. The same formula holds for the random vector $\mathbf{Y}$. This proves the result applying Theorem 3.3.

The order $\preceq_{t v m}$ is not preserved by marginalization as we show in the following example.

Example 3.16. Let $\mathbf{X}$ and $\mathbf{Y}$ be random vectors with distributions degenerated at the points $(0,0,1,1)$ and $(10,10,0,1)$, respectively. Clearly $(0,0,1,1) \leq_{h^{-1}}(10,10,0,1)$, and so $\mathbf{X} \preceq_{\text {tvm }} \mathbf{Y}$.

Let us consider the random vectors $\left(X_{3}, X_{4}\right)$ and $\left(Y_{3}, Y_{4}\right)$. Consider $Q_{(1,1)}$ the quadrant set of the point $(1,1)$ with respect to the partial order $\leq_{h^{-1}}$ on $\mathbb{R}^{2}$. It holds that $(0,1) \notin Q_{(1,1)}$, and so $P\left(\left(X_{3}, X_{4}\right) \in Q_{(1,1)}\right)=1>$ $P\left(\left(Y_{3}, Y_{4}\right) \in Q_{(1,1)}\right)=0$. Thus $\left(X_{3}, X_{4}\right) \preceq_{\text {tvm }}\left(Y_{3}, Y_{4}\right)$ is false (see iii) in Proposition 3.8).

The above example also implies that the stochastic order $\preceq_{t v m}$ is not closed under identical concatenation.

\subsection{Connections with other stochastic orders and ex- amples}

In this subsection we analyze possible relations of the time value of money order with other multivariate stochastic orders. Examples of some parametric families ordered in the new order in accordance with their parameters, are also proposed.

Proposition 3.17. The multivariate stochastic order $\preceq_{s t}$ implies the order $\preceq_{t v m}$.

Proof. Observe that the maximal generator of the order $\preceq_{s t}$ (when the weight function is $b=1$ ) is composed of the increasing and bounded mappings, which contains the maximal generator of the stochastic order $\preceq_{\text {tvm }}$. 
The stochastic order $\preceq_{t v m}$ is weaker than the stochastic order $\preceq_{s t}$ as the following example shows.

Example 3.18. Let $\mathrm{X}$ be a bidimensional random vector with distribution degenerated at the point $(1,-1)$. Let $\mathbf{Y}$ be a random vector with distribution degenerated at the point $(2,-2)$. Note that $(1,-1) \leq_{h^{-1}}(2,-2)$. Thus, given any $f \in \mathcal{F}_{b}$ we have that $f((2,-2)) \geq f((1,-1))$. As a consequence, $\mathbf{X} \preceq$ tvm $\mathbf{Y}$. On the other hand, the relation $\mathbf{X} \preceq_{s t} \mathbf{Y}$ is false.

Proposition 3.19. Let $\boldsymbol{X}=\left(X_{1}, \ldots, X_{n}\right)$ and $\boldsymbol{Y}=\left(Y_{1}, \ldots, Y_{n}\right)$ be random vectors such that $\boldsymbol{X} \preceq_{\text {tvm }} \boldsymbol{Y}$. If $E \boldsymbol{X}=E \boldsymbol{Y}$ then $\boldsymbol{X} \sim_{s t} \boldsymbol{Y}$.

Proof. Note that $E \mathbf{X}=E \mathbf{Y}$ implies that $E\left(h^{-1}(\mathbf{X})\right)=E\left(h^{-1}(\mathbf{Y})\right)$. This and $h^{-1}(\mathbf{X}) \preceq_{s t} h^{-1}(\mathbf{Y})$ lead to $h^{-1}(\mathbf{X}) \sim_{s t} h^{-1}(\mathbf{Y})$, which is the same as $\mathbf{X} \sim_{s t} \mathbf{Y}$ since $h^{-1}$ is bijective.

Proposition 3.20. The order $\preceq_{\text {tvm }}$ implies the order $\preceq_{1}$.

Proof. Let $\mathbf{X}=\left(X_{1}, \ldots, X_{n}\right)$ and $\mathbf{Y}=\left(Y_{1}, \ldots, Y_{n}\right)$ be random vectors such that $\mathbf{X} \preceq_{t v m} \mathbf{Y}$. Theorem 3.3 reads that $h^{-1}(\mathbf{X}) \preceq_{s t} h^{-1}(\mathbf{Y})$. Given $v \geq 0$ in $\mathbb{R}^{n}$, let $g_{v}: \mathbb{R}^{n} \rightarrow \mathbb{R}$ with $g_{v}(x)=\langle v, x\rangle$ for all $x \in \mathbb{R}^{n}$, where $\langle\cdot, \cdot\rangle$ denotes the inner product in $\mathbb{R}^{n}$. Note that $g_{v}\left(h^{-1}(\mathbf{X})\right) \preceq_{s t} g_{v}\left(h^{-1}(\mathbf{Y})\right)$ since $g_{v}$ is increasing.

Consider $\phi=\left(\phi_{1}, \phi_{2}, \ldots, \phi_{n}\right) \in \mathbb{R}^{n}$ with $1 \geq \phi_{1} \geq \phi_{2} \ldots \geq \phi_{n} \geq 0$, and $v \in \mathbb{R}^{n}$ such that $v_{i}=\phi_{i}-\phi_{i+1}$ for all $1 \leq i \leq n-1$ and $v_{n}=\phi_{n}$. We have that $g_{v}\left(h^{-1}(\mathbf{X})\right)=\phi_{1} X_{1}+\ldots+\phi_{n} X_{n}$ and $g_{v}\left(h^{-1}(\mathbf{Y})\right)=\phi_{1} Y_{1}+\ldots+\phi_{n} Y_{n}$. As a consequence, $\mathbf{X} \preceq_{1} \mathbf{Y}$ holds.

The converse of Proposition 3.20 is not true in general as the following example shows.

Example 3.21. Consider a 2-years investment where $x_{1}$ and $x_{2}$ are the annual cash flows at the end of the first and second year respectively. Suppose that there exists a bonus of two monetary units when at least one of the cumulative cash flows at the end of each year, is greater than one monetary unit. Assume that the bonus is paid at the end of the second year. The present value of the investment is

$$
f_{r}\left(x_{1}, x_{2}\right)=\frac{x_{1}}{1+r}+\frac{x_{2}}{(1+r)^{2}}+\frac{2 I_{[1,+\infty)}\left(\max \left\{x_{1}, x_{1}+x_{2}\right\}\right)}{(1+r)^{2}},
$$


where $r \geq 0$ is the annual interest rate and $I_{[1,+\infty)}$ is the indicator function of $[1,+\infty)$.

It is not hard to prove that $f_{r}$ belongs to $\mathcal{F}$ for any $r \geq 0$.

Consider the random vectors $\mathbf{X}$ and $\mathbf{Y}$ with supports $\{(1,0),(0,1),(1,-1)\}$ and $\{(3 / 4,0),(1,1),(2,-1)\}$ respectively, all the points with the same probability. When $r=0, E\left(f_{0}(\mathbf{X})\right)=8 / 3$ and $E\left(f_{0}(\mathbf{Y})\right)=31 / 12$, which implies that $\mathbf{X} \preceq_{\text {tvm }} \mathbf{Y}$ is not true. On the other hand, it is not difficult to prove that $\mathbf{X} \preceq_{1} \mathbf{Y}$.

Remark 3.22. Let $\mathbf{X}=\left(X_{1}, \ldots, X_{n}\right)$ and $\mathbf{Y}=\left(Y_{1}, \ldots, Y_{n}\right)$ be random vectors such that $\mathbf{X} \preceq^{1} \mathbf{Y}$, then $\mathbf{X} \preceq^{2} \mathbf{Y}$ holds (see Scarsini (1986)). As a consequence, the order $\preceq_{t v m}$ implies the order $\preceq_{2}$, but the converse is not true.

Remark 3.23. There are not specific relations between the orders $\preceq_{t v m}$ and $\preceq_{u o}$, and the orders $\preceq_{\text {tvm }}$ and $\preceq_{l o}$.

Example 3.18 shows that neither $\preceq_{u o}$ nor $\preceq_{l o}$ are implied by the order $\preceq_{\text {tvm }}$ since in that case $\mathbf{X} \preceq_{u o} \mathbf{Y}$ and $\mathbf{X} \preceq_{l o} \mathbf{Y}$ are false. Note that $\bar{F}_{\mathbf{X}}(1 / 2,-3 / 2)>\bar{F}_{\mathbf{Y}}(1 / 2,-3 / 2)$ and $F_{\mathbf{X}}(2,-2)<F_{\mathbf{Y}}(2,-2)$.

Now consider the random vectors $\mathbf{X}$ and $\mathbf{Y}$ with supports $\{(0,0),(2,-1)$, $(1,-2)\}$ and $\{(3,0),(0,-1),(2,-2)\}$ respectively, all the points with the same probability.

It is easy to see that $\mathbf{X} \preceq_{u o} \mathbf{Y}$. Consider the set $M=\left\{(x, y) \in \mathbb{R}^{2} \mid x \geq\right.$ $0, y+2 x \geq 0\}$. It is not hard to prove that $M$ is a measurable upper set for the partial order $\leq_{h^{-1}}$ on $\mathbb{R}^{2}$. Proposition $3.8 i v$ ) ensures that $\mathbf{X} \preceq_{t v m} \mathbf{Y}$ is false.

Let us take the random vectors $\mathbf{X}$ and $\mathbf{Y}$ with supports $\{(0,0),(1,1)\}$ and $\{(1,0),(0,1)\}$ respectively, all the points with the same probability. The relation $\mathbf{X} \preceq_{l o} \mathbf{Y}$ holds. Moreover, $h^{-1}(\mathbf{X}) \preceq_{s t} h^{-1}(\mathbf{Y})$ is false and so is $\mathbf{X} \preceq_{t v m} \mathbf{Y}$.

Multivariate normal and $t$ distributions are mostly adopted in practice by investors in our framework. We analyze the time value of money order with those distributions.

Now, we provide sufficient and necessary conditions for random vectors with normal distribution to be ordered in the stochastic order $\preceq_{t v m}$.

Proposition 3.24. Let $\boldsymbol{X}=\left(X_{1}, \ldots, X_{n}\right)$ and $\boldsymbol{Y}=\left(Y_{1}, \ldots, Y_{n}\right)$ be random vectors with normal distribution, $\boldsymbol{X} \sim_{s t} N(\mu, \Sigma)$ and $\boldsymbol{Y} \sim_{s t} N\left(\mu^{\prime}, \Sigma^{\prime}\right)$. Then, $\boldsymbol{X} \preceq_{\text {tvm }} \boldsymbol{Y}$ if and only if 
i) $\mu_{1}+\mu_{2}+\cdots+\mu_{k} \leq \mu_{1}^{\prime}+\mu_{2}^{\prime}+\cdots+\mu_{k}^{\prime}$ for any $1 \leq k \leq n$,

ii) $\Sigma=\Sigma^{\prime}$

Proof. By Theorem 3.3, $\mathbf{X} \preceq_{t v m} \mathbf{Y}$ if and only if $h^{-1}(\mathbf{X}) \preceq_{s t} h^{-1}(\mathbf{Y})$.

On the other hand $h^{-1}(\mathbf{X}) \sim_{s t} N\left(h^{-1}(\mu), H^{-1} \Sigma\left(H^{-1}\right)^{t}\right)$ and $h^{-1}(\mathbf{Y}) \sim_{s t}$ $N\left(h^{-1}\left(\mu^{\prime}\right), H^{-1} \Sigma^{\prime}\left(H^{-1}\right)^{t}\right)$, where $H$ is the matrix associated with $h$ with respect to $\left\{e_{1}, \ldots, e_{n}\right\}$.

By Theorem 3.3.13 in Müller and Stoyan (2002), $h^{-1}(\mathbf{X}) \preceq_{s t} h^{-1}(\mathbf{Y})$ if and only if $h^{-1}(\mu) \leq h^{-1}\left(\mu^{\prime}\right)$ and $H^{-1} \Sigma\left(H^{-1}\right)^{t}=H^{-1} \Sigma^{\prime}\left(H^{-1}\right)^{t}$.

The condition $h^{-1}(\mu) \leq h^{-1}\left(\mu^{\prime}\right)$ is the same as $\left.i\right)$. Since $H$ is regular, $H^{-1} \Sigma\left(H^{-1}\right)^{t}=H^{-1} \Sigma^{\prime}\left(H^{-1}\right)^{t}$ is equivalent to $\Sigma=\Sigma^{\prime}$, which concludes the proof.

The case of the multivariate $t$ distribution is analyzed in the following results.

Lemma 3.25. Let $X$ and $Y$ be random variables with $t$ distribution with freedom degrees $\nu$ and $\nu^{\prime}$, respectively. Let $a, a^{\prime}>0$ and $b, b^{\prime} \in \mathbb{R}$. Then, $a X+b \preceq_{s t} a^{\prime} Y+b^{\prime}$ if and only if $\nu=\nu^{\prime}, a=a^{\prime}$ and $b \leq b^{\prime}$.

Proof. Let us suppose that $a X+b \preceq_{s t} a^{\prime} Y+b^{\prime}$. Then, $E(a X+b) \leq E\left(a^{\prime} Y+\right.$ $\left.b^{\prime}\right)$, and so $b \leq b^{\prime}$.

Let us see that $\nu=\nu^{\prime}$. Note that the density mappings of $a X+b$ and $a^{\prime} Y+b^{\prime}$ are $f_{a X+b}(x)=f_{X}\left(\frac{x-b}{a}\right) \frac{1}{a}$ and $f_{a^{\prime} Y+b^{\prime}}(x)=f_{Y}\left(\frac{x-b^{\prime}}{a^{\prime}}\right) \frac{1}{a^{\prime}}$ for any $x \in \mathbb{R}$.

Suppose that $\nu>\nu^{\prime}$. It can be seen that $\lim _{t \rightarrow-\infty} \frac{f_{a X+b}(t)}{f_{a^{\prime} Y+b^{\prime}}(t)}=0$. As a consequence, there exist $m \in \mathbb{R}$ such that $f_{a X+b}(t) \leq f_{a^{\prime} Y+b^{\prime}}(t)$ for any $t \leq m$. Hence, $P(a X+b \leq m)<P\left(a^{\prime} Y+b^{\prime} \leq m\right)$, which is a contradiction with $a X+b \preceq_{s t} a^{\prime} Y+b^{\prime}$.

The case $\nu<\nu^{\prime}$ can be analyzed in a similar way. Therefore $\nu=\nu^{\prime}$. Thus, $X \sim_{s t} Y$.

Let us see that $a=a^{\prime}$. Suppose that $a<a^{\prime}$. Let $m_{1} \in \mathbb{R}$ with $\frac{m_{1}-b}{a} \leq$ $\frac{m_{1}-b^{\prime}}{a^{\prime}}$. We have that $P\left(a X+b \leq m_{1}\right)=P\left(X \leq \frac{m_{1}-b}{a}\right)=P\left(Y \leq \frac{m_{1}-b}{a}\right) \leq$ $P\left(Y \leq \frac{m_{1}-b^{\prime}}{a^{\prime}}\right)=P\left(a^{\prime} Y+b^{\prime} \leq m_{1}\right)$, which is a contradiction with $a X+b \preceq s t$ $a^{\prime} Y+b^{\prime}$. The case $a>a^{\prime}$ can be studied in the same way taking $m_{2} \in \mathbb{R}$ with $\frac{m_{2}-b}{a}<\frac{m_{2}-b^{\prime}}{a^{\prime}}$. Therefore, $a=a^{\prime}$.

The converse is trivial. 
A random vector $\mathbf{X}=\left(X_{1}, \ldots, X_{n}\right)$ follows a multivariate $t$ distribution with freedom degrees $\nu$, mean vector $\mu$ and positive definite symmetric matrix parameter $\Sigma$, if its density function is

$$
f_{\mathbf{X}}(x)=\frac{\Gamma\left(\frac{\nu+n}{2}\right)}{\Gamma\left(\frac{\nu}{2}\right)} \frac{1}{(\nu \pi)^{n / 2}} \frac{1}{\sqrt{\operatorname{det} \Sigma}}\left(1+\frac{1}{\nu}(x-\mu)^{T} \Sigma^{-1}(x-\mu)\right)^{-\frac{n+\nu}{2}}
$$

for any $x \in \mathbb{R}^{n}$ (see, for instance, Roth (2013) for the multivariate $t$ distribution and its properties).

Proposition 3.26. Let $\boldsymbol{X}=\left(X_{1}, \ldots, X_{n}\right)$ and $\boldsymbol{Y}=\left(Y_{1}, \ldots, Y_{n}\right)$ be random vectors with $t$ distribution with freedom degrees $\nu$ and $\nu^{\prime}$, mean vectors $\mu$ and $\mu^{\prime}$ and matrices parameter $\Sigma$ and $\Sigma^{\prime}$, respectively. It holds that $\boldsymbol{X} \preceq_{\text {st }} \boldsymbol{Y}$ if and only if

i) $\mu_{i} \leq \mu_{i}^{\prime}$ for any $1 \leq i \leq n$,

ii) $\nu=\nu^{\prime}$,

iii) $\Sigma=\Sigma^{\prime}$.

Proof. Clearly enough, if $\nu=\nu^{\prime}, \Sigma=\Sigma^{\prime}$ and $\mu_{i} \leq \mu_{i}^{\prime}$ for any $1 \leq i \leq n$, then $\mathbf{X} \preceq_{s t} \mathbf{Y}$. Note that $\mathbf{Y} \sim_{s t} \mathbf{X}+\mu^{\prime}-\mu$.

Now, assume that $\mathbf{X} \preceq_{s t} \mathbf{Y}$.

Thus, $X_{i} \preceq_{s t} Y_{i}$ for any $1 \leq i \leq n$. By the properties of the multivariate $t$ distribution, it holds that $X_{i} \sim_{s t} \sqrt{(\Sigma)_{i i}} W+\mu_{i}$, where $W$ is a random variable which follows $t$ distribution with freedom degrees $\nu$ and $Y_{i} \sim_{s t} \sqrt{\left(\Sigma^{\prime}\right)_{i i}} W^{\prime}+\mu_{i}^{\prime}$, where $W^{\prime}$ is a random variable which follows $t$ distribution with freedom degrees $\nu^{\prime}$. Therefore, $\sqrt{(\Sigma)_{i i}} W+\mu_{i} \preceq s t \sqrt{\left(\Sigma^{\prime}\right)_{i i}} W^{\prime}+\mu_{i}^{\prime}$.

By Lemma 3.25, we obtain that $\nu=\nu^{\prime},(\Sigma)_{i i}=\left(\Sigma^{\prime}\right)_{i i}$ and $\mu_{i} \leq \mu_{i}^{\prime}$.

In a similar way, $\mathbf{X} \preceq_{s t} \mathbf{Y}$ implies that $X_{i}+X_{j} \preceq_{s t} Y_{i}+Y_{j}$ for any $1 \leq$ $i, j \leq n$ with $i \neq j$. The properties of the multivariate $t$ distribution imply that $X_{i}+X_{j} \sim_{s t} \sqrt{(\Sigma)_{i i}+(\Sigma)_{j j}+2(\Sigma)_{i j}} W+\mu_{i}+\mu_{j}$ where $W$ is a $t$ random variable with freedom degrees $\nu$ and $Y_{i}+Y_{j} \sim_{s t} \sqrt{\left(\Sigma^{\prime}\right)_{i i}+\left(\Sigma^{\prime}\right)_{j j}+2\left(\Sigma^{\prime}\right)_{i j}} W^{\prime}+$ $\mu_{i}^{\prime}+\mu_{j}^{\prime}$ where $W^{\prime}$ is a $t$ random variable with freedom degrees $\nu^{\prime}$.

Lemma 3.25 implies that $(\Sigma)_{i i}+(\Sigma)_{j j}+2(\Sigma)_{i j}=\left(\Sigma^{\prime}\right)_{i i}+\left(\Sigma^{\prime}\right)_{j j}+2\left(\Sigma^{\prime}\right)_{i j}$. Thus, $(\Sigma)_{i j}=\left(\Sigma^{\prime}\right)_{i j}$, which concludes the proof.

Proposition 3.27. Let $\boldsymbol{X}=\left(X_{1}, \ldots, X_{n}\right)$ and $\boldsymbol{Y}=\left(Y_{1}, \ldots, Y_{n}\right)$ be random vectors with $t$ distribution with freedom degrees $\nu$ and $\nu^{\prime}$, mean vectors $\mu$ and $\mu^{\prime}$ and matrix parameter $\Sigma$ and $\Sigma^{\prime}$, respectively. Then, $\boldsymbol{X} \preceq_{t v m} \boldsymbol{Y}$ if and only if 
i) $\mu_{1}+\mu_{2}+\cdots+\mu_{k} \leq \mu_{1}^{\prime}+\mu_{2}^{\prime}+\cdots+\mu_{k}^{\prime}$ for any $1 \leq k \leq n$,

ii) $\nu=\nu^{\prime}$,

iii) $\Sigma=\Sigma^{\prime}$.

Proof. Theorem 3.3 says that $\mathbf{X} \preceq_{\text {tvm }} \mathbf{Y}$ is equivalent to $h^{-1}(\mathbf{X}) \preceq_{s t} h^{-1}(\mathbf{Y})$. The properties of the multivariate $t$ distribution assure that $h^{-1}(\mathbf{X})$ and $h^{-1}(\mathbf{Y})$ follow $t$ distributions with freedom degrees $\nu$ and $\nu^{\prime}$, mean vectors $H^{-1} \mu$ and $H^{-1} \mu^{\prime}$ and matrices parameter $H^{-1} \Sigma\left(H^{-1}\right)^{t}$ and $H^{-1} \Sigma^{\prime}\left(H^{-1}\right)^{t}$, respectively, where $H$ is the matrix associated with $h$ with respect to $\left\{e_{1}, \ldots, e_{n}\right\}$.

Now, by Proposition 3.27, we obtain that $\mathbf{X} \preceq_{t v m} \mathbf{Y}$ is equivalent to $\nu=$ $\nu^{\prime},\left(H^{-1} \mu\right)_{i} \leq\left(H^{-1} \mu^{\prime}\right)_{i}$ for any $1 \leq i \leq n$, and $H^{-1} \Sigma\left(H^{-1}\right)^{t}=H^{-1} \Sigma^{\prime}\left(H^{-1}\right)^{t}$.

Note that $\left(H^{-1} \mu\right)_{i} \leq\left(H^{-1} \mu^{\prime}\right)_{i}$ for any $1 \leq i \leq n$, is the same as $\mu_{1}+$ $\mu_{2}+\cdots+\mu_{k} \leq \mu_{1}^{\prime}+\mu_{2}^{\prime}+\cdots+\mu_{k}^{\prime}$ for any $1 \leq k \leq n$, and since $H$ is regular, $H^{-1} \Sigma\left(H^{-1}\right)^{t}=H^{-1} \Sigma^{\prime}\left(H^{-1}\right)^{t}$ is equivalent to $\Sigma=\Sigma^{\prime}$, which proves the proposition.

Other parametric families are analyzed below.

Consider the probability distribution given by the following density with parameters $\left(\theta_{0}, \theta_{1}, \ldots, \theta_{n-1}\right)$, where $\theta_{i}>0,0 \leq i \leq n-1$,

$$
g\left(x_{1}, x_{2}, \ldots, x_{n}\right)=n ! \prod_{j=0}^{n-1}\left(\theta_{j}^{-1} \exp \left\{-(n-j) \theta_{j}^{-1}\left(x_{j+1}-x_{j}\right)\right\}\right)
$$

with $x_{0}=0<x_{1} \leq \ldots \leq x_{n}$ (see Fernández-Ponce and Suárez-Lloréns $(2003))$.

Proposition 3.28. Let $\boldsymbol{X}=\left(X_{1}, \ldots, X_{n}\right)$ and $\boldsymbol{Y}=\left(Y_{1}, \ldots, Y_{n}\right)$ be random vectors distributed in accordance with the previous density function and with parameters $\left(\theta_{0}, \theta_{1}, \ldots, \theta_{n-1}\right)$ and $\left(\theta_{0}^{\prime}, \theta_{1}^{\prime}, \ldots, \theta_{n-1}^{\prime}\right)$ respectively. Let $\mu_{i}=$ $\theta_{i}^{\prime} / \theta_{i}, 0 \leq i \leq n-1$. If $\mu_{i} \geq 1$ for all $0 \leq i \leq n-1$ and $j \mu_{i}-(j-1) \mu_{i+1} \geq 1$ with $0 \leq i \leq n-2$ and $0 \leq j \leq n-i$, then $\boldsymbol{X} \preceq$ tvm $\boldsymbol{Y}$. 
Proof. It holds that $A \mathbf{X} \sim_{s t} \mathbf{Y}$ with

$$
A=\left(\begin{array}{cccccc}
\frac{\theta_{0}^{\prime}}{\theta_{0}} & 0 & 0 & 0 & \ldots & 0 \\
\frac{\theta_{0}}{\theta_{0}}-\frac{\theta_{1}^{\prime}}{\theta_{1}} & \frac{\theta_{1}^{\prime}}{\theta_{1}} & 0 & 0 & \ldots & 0 \\
\frac{\theta_{0}^{\prime}}{\theta_{0}}-\frac{\theta_{1}^{\prime}}{\theta_{1}} & \frac{\theta_{1}^{\prime}}{\theta_{1}}-\frac{\theta_{2}^{\prime}}{\theta_{2}} & \frac{\theta_{2}^{\prime}}{\theta_{2}} & 0 & \ldots & 0 \\
\frac{\theta_{0}^{\prime}}{\theta_{0}}-\frac{\theta_{1}^{\prime}}{\theta_{1}} & \frac{\theta_{1}^{\prime}}{\theta_{1}}-\frac{\theta_{2}^{\prime}}{\theta_{2}} & \frac{\theta_{2}^{\prime}}{\theta_{2}}-\frac{\theta_{3}^{\prime}}{\theta_{3}} & \frac{\theta_{3}^{\prime}}{\theta_{3}} & \ldots & 0 \\
\vdots & \vdots & \vdots & \vdots & \vdots & \vdots \\
\frac{\theta_{0}^{\prime}}{\theta_{0}}-\frac{\theta_{1}^{\prime}}{\theta_{1}} & \frac{\theta_{1}^{\prime}}{\theta_{1}}-\frac{\theta_{2}^{\prime}}{\theta_{2}} & \frac{\theta_{2}^{\prime}}{\theta_{2}}-\frac{\theta_{3}^{\prime}}{\theta_{3}} & \frac{\theta_{3}^{\prime}}{\theta_{3}}-\frac{\theta_{4}^{\prime}}{\theta_{4}} & \ldots & \frac{\theta_{n-1}^{\prime}}{\theta_{n-1}}
\end{array}\right) .
$$

Note that

$$
A \mathbf{X}=\left(\begin{array}{c}
\mu_{0} X_{1} \\
\left(\mu_{0}-\mu_{1}\right) X_{1}+\mu_{1} X_{2} \\
\left(\mu_{0}-\mu_{1}\right) X_{1}+\left(\mu_{1}-\mu_{2}\right) X_{2}+\mu_{2} X_{3} \\
\left(\mu_{0}-\mu_{1}\right) X_{1}+\left(\mu_{1}-\mu_{2}\right) X_{2}+\left(\mu_{2}-\mu_{3}\right) X_{3}+\mu_{3} X_{4} \\
\vdots \\
\vdots \\
\left(\mu_{0}-\mu_{1}\right) X_{1}+\left(\mu_{1}-\mu_{2}\right) X_{2}+\cdots+\left(\mu_{n-2}-\mu_{n-1}\right) X_{n-1}+\mu_{n-1} X_{n}
\end{array}\right) .
$$

It holds that $\mathbf{X} \leq_{h^{-1}} A \mathbf{X}$ in accordance with the conditions of the parameters $\mu_{i}$. Now $i i$ ) in Proposition 3.8 leads to the result.

Let us consider the multivariate density given by

$$
g\left(x_{1}, x_{2}, \ldots, x_{n}\right)=\frac{\Gamma(k+n)}{\Gamma(k)}\left(1+\sum_{j=1}^{n} \alpha_{j} x_{j}\right)^{-(k+n)} \prod_{j=1}^{n} \alpha_{j}
$$

with $x_{j}>0, k>0, \alpha_{j}>0,1 \leq j \leq n$ (see, for instance, Johnson and Kotz (1972), page 289).

Proposition 3.29. Let $\boldsymbol{X}=\left(X_{1}, \ldots, X_{n}\right)$ and $\boldsymbol{Y}=\left(Y_{1}, \ldots, Y_{n}\right)$ be random vectors whose distributions are given by the above densities with parameters $\left(\alpha_{1}, \ldots, \alpha_{n}, k\right)$ and $\left(\alpha_{1}^{\prime}, \ldots, \alpha_{n}^{\prime}, k\right)$ respectively. If $\frac{\alpha_{i}}{\alpha_{i}^{\prime}} \geq 1$ for any $1 \leq i \leq n$, then $\boldsymbol{X} \preceq_{\text {tvm }} \boldsymbol{Y}$.

Proof. Let $A=\operatorname{diag}\left(\frac{\alpha_{1}}{\alpha_{1}^{\prime}}, \frac{\alpha_{2}}{\alpha_{2}^{\prime}}, \ldots, \frac{\alpha_{n}}{\alpha_{n}^{\prime}}\right)$. It is not hard to prove that $A \mathbf{X} \sim_{s t} \mathbf{Y}$. Clearly $\mathbf{X} \leq_{h^{-1}} A \mathbf{X}$, which implies the result. 


\section{Conclusions}

In this paper we have introduced a tool to compare investments affected by the time value of money, as those involving cash flows at different moments in time. That tool is based on an integral stochastic order whose generator is given by the mappings which reflect the effects of the time value of money. Different characterizations of the order are proved like that based on the usual multivariate stochastic order which facilitates the analysis of the order. The maximal generator of the order is obtained. The new stochastic order can be seen as an order generated by a (non-stochastic) partial order on $\mathbb{R}^{n}$ and the class mappings which preserve the partial order. Moreover, multiple properties of the order and relations with other stochastic orders are also stated.

Acknowledgements. The authors would like to thank the referees and the editor for their interesting comments and suggestions which have improved the manuscript. The authors are indebted to the Spanish Ministry of Science and Innovation and Principado de Asturias since this research is financed by Grants MTM2013-45588-C3-1-P, MTM2015-63971-P, FC-15-GRUPIN14-101 and FC-15-GRUPIN14-142.

\section{References}

Belzunce, F., Martínez-Riquelme, C., Mulero, J., 2016. An Introduction to Stochastic Orders. Amsterdam: Elsevier/Academic.

Brealey, R.A., Myers, S.C., Allen, F., 2014. Principles of Corporate Finance, 11/edition. New York: Mc Graw Hill.

Crosson, S.V., Needles, B.E., 2010. Managerial Accounting, 9th Ed. Boston: Houghton Mifflin Company.

Dhaene, J., Goovaerts, M., Vanmaele, M., Van Weert, K., 2012. Convex order approximations in the case of cash flows of mixed signs. Insurance: Mathematics \& Economics 51, 249-256.

De Schepper, A., Goovaerts, M., Dhaene, J., Kaas, R., Vyncke, D., 2002. Bounds for present value functions with stochastic interest rates and stochastic volatility. 5th IME Conference (University Park, PA, 2001). Insurance: Mathematics \& Economics 31, 87-103. 
Fernández-Ponce, J.M., Suárez-Lloréns, A., 2003. A multivariate dispersion ordering based on quantiles more widely separated. Journal of Multivariate Analysis 85, 40-53.

Halmos, P.R., 1950. Measure Theory. New York: D. Van Nostrand Company, Inc.

Johnson, N.L., Kotz, S., 1972. Distributions in statistics: continuous multivariate distributions. New York-London-Sydney: John Wiley \& Sons, Inc.

Kamae, T., Krengel, U., O'Brien, G.L., 1977. Stochastic inequalities on partially ordered spaces. Annals of Probability 5, 899-912.

Kamae, T., Krengel, U., 1978. Stochastic partial ordering. Annals of Probability 6, 1044-1049.

López-Díaz, M.C., López-Díaz, M., 2012. When is an integral stochastic order generated by a poset? Journal of Inequalities and Applications 265, 8 pp.

López-Díaz, M.C., López-Díaz, M., 2013a. A note on the family of extremality stochastic orders. Insurance: Mathematics \& Economics 53, 230-236.

López-Díaz, M.C., López-Díaz, M., 2013b. On order-isomorphisms of stochastic orders generated by partially ordered sets with applications to the analysis of chemical components of seaweeds. MATCH Communications in Mathematical and in Computer Chemistry 69, 463-486.

Müller, A., 1997. Stochastic orders generated by integrals: a unified study. Advances in Applied Probability 29, 414-428.

Müller, A., Stoyan, D., 2002. Comparison Methods for Stochastic Models and Risks. Chichester: John Wiley \& Sons.

Neggers, J., Kim, H.S., 1998. Basic posets. Singapore: World Scientific Publishing.

Roth, M., 2013. On the Multivariate $t$ Distribution. Technical report from Automatic Control at Linköpings universitet. Report no.: LiTH-ISY-R-3059.

Scarsini, M., 1986. Comparison of random cash flows. IMA Journal of Mathematics in Management 1, 25-32.

Schröder, B.S.W., 2003. Ordered sets. An introduction. Boston-Basel-Berlin: Birkhäuser.

Shaked, M., Shanthikumar, J.G., 2007. Stochastic Orders. New York: Springer. 
Strassen, V., 1965. The existence of probability measures with given marginals. Annals of Mathematical Statistics 36, 423-439.

Tretyakov, M.V., 2013. Introductory course on financial mathematics. London: Imperial College Press. 